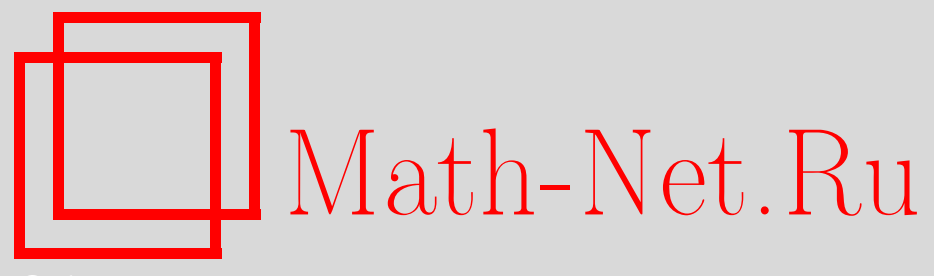

С. Е. Клевцов, К вертекс-операторной конструкции квантовых аффинных алгебр, ТМФ, 2008, том 154, номер 2, 240-248

DOI: https://doi.org/10.4213/tmf6165

Использование Общероссийского математического портала Math-Net.Ru подразумевает, что вы прочитали и согласны с пользовательским соглашением http://www . mathnet.ru/rus/agreement

Параметры загрузки:

IP : 52.205 .19 .152

26 апреля 2023 г., 17:06:16

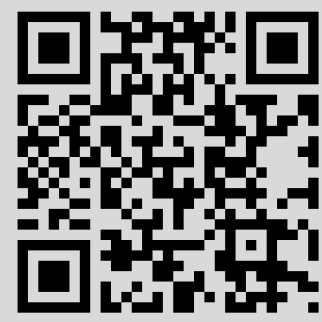




\title{
К ВЕРТЕКС-ОПЕРАТОРНОЙ КОНСТРУКЦИИ КВАНТОВЫХ АФФИННЫХ АЛГЕБР
}

\begin{abstract}
Описана конструкция квантовых аффинных алгебр вершинными операторами в теории свободных полей. Доказаны тождества Серра для борелевских подалгебр квантовых аффинных алгебр, в частности случай $\widehat{s l}_{2}$ рассмотрен детально. Также построены генераторы, соответствующие положительным корням $\widehat{s l}_{2}$.
\end{abstract}

Ключевые слова: квантовые группы, квантовые аффинные алгебры, теория свободных полей, минимальные модели, соотношения Серра.

\section{1. ВВЕДЕНИЕ}

Алгебры вершинных (вертексных) операторов [1]-[3] в теории свободных полей [4]-[6] обладают богатой математической структурой. Хорошо известна их связь с теорией представлений аффинных алгебр Ли [7], а также с теорией квантовых групп [8]. Различные аспекты вертекс-операторных конструкций квантовых групп также изучались в работах [9], [10].

В этой статье мы изучаем вертекс-операторную конструкцию квантовых борелевских подалгебр конечномерных и аффинных алгебр в теории свободных полей $\varphi^{i}$, $i=1,2, \ldots, n$. Теория свободных полей описывается действием

$$
S=\int d^{2} z\left(\partial \varphi^{i} \bar{\partial} \varphi^{i}+i \alpha_{0} R \rho^{i} \varphi^{i}\right),
$$

где $R$ - двумерная кривизна фоновой метрики, $\alpha_{0}$ - экранирующий заряд, $\rho^{i}-$ некоторый постоянный вектор. Пусть $\varphi^{i}$ принимают значения на некотором торе. Решетка корней, соответствующая тору, порождает некоторую алгебру Ли $g$ [2]. Мы рассматриваем вершинные операторы конформной размерности 1 и изучаем их коммутационные соотношения. Строго говоря, если алгебра Ли $g$ имеет некоторое разложение Картана $g=n_{-} \oplus h \oplus n_{+}$, то вершинные операторы, соответствующие простым корням, удовлетворяют соотношениям $U_{q}\left(n_{+}\right)$с некоторым параметром деформации $q=q_{+}$. Аналогично можно получить квантовую группу $U_{q}\left(n_{-}\right)$ (с некоторым другим параметром деформации $q_{-}$).

${ }^{*}$ Институт теоретической и экспериментальной физики; Московский государственный университет им. М.В.Ломоносова, Москва, Россия. E-mail: klevtsov@itep.ru 
Конструкции подобного рода для конечномерных алгебр типа $A D E$ были предложены в работе [6] и изучены в [9] (см. также [10]). Целью настоящей работы является обобщение этой конструкции на случай квантовых аффинных алгебр (рассмотрены также и конечномерные алгебры). Мы доказываем соотношения Серра и приводим явные формулы для генераторов в аффинном случае. Отметим, что соотношения Серра в аффинных квантовых алгебрах изучались также в рабо$\operatorname{Tax}[11],[12]$.

В классическом пределе $\alpha_{0} \rightarrow 0$ вершинные операторы в теории с действием (1) должны сводиться к известным формулам [2]. В подходе, примененном в данной работе, предел $\alpha_{0} \rightarrow 0$ может быть получен явно (ср. с [9], [10], [13], где этот предел неочевиден).

Существует тесная связь между конформными минимальными моделями и квантовой конформной теорией Тоды при специальных значениях параметров. Можно полагать, что предложенная в этой работе конструкция может быть полезна для понимания квантования конформной аффинной теории Тоды [14].

Работа организована следующим образом. В разделе 2 описана конструкция $U_{q}\left(s l_{3}\right)$. В разделе 3 доказаны тождества Серра в этом случае. Конструкция квантовой аффинной алгебры $U_{q}\left(\widehat{s l}_{2}\right)$ представлена в разделе 4 ; детали доказательства тождеств Серра и формулы для генераторов приведены в приложении. Отметим также, что доказательство соотношений Серра для $U_{q}\left(s l_{3}\right)$ полностью переносится на случай произвольной алгебры типа $A D E$, т.е. представленная конструкция обобщается на случай произвольной алгебры данного типа.

\section{2. КОНСТРУКцИЯ $U_{q}\left(s l_{3}\right)$}

Тензор энергии-импульса, соответствующий действию (1), дается выражением

$$
T(z)=-\frac{1}{2}(\partial \varphi(z), \partial \varphi(z))+i \alpha_{0}\left(\rho, \partial^{2} \varphi(z)\right)
$$

где $(\cdot, \cdot)$ обозначает скалярное произведение, т.е. $\varphi^{i} \varphi^{i}=(\varphi, \varphi)$. Тензор $T(z)$ генерирует алгебру Вирасоро с центральным зарядом $c=n-12 \alpha_{0}^{2}(\rho, \rho)$. Рассмотрим следующие вершинные операторы, проинтегрированные по подходящему контуру:

$$
\int d z V_{\alpha}^{ \pm}(\varphi)=\int d z: e^{i \alpha_{ \pm}(\alpha, \varphi)}:
$$

где : . . : обозначает нормальное упорядочение, а $\alpha_{ \pm}-$некоторые константы, значения которых определяются из условия равенства единице конформной размерности оператора $\Delta$ :

$$
\begin{aligned}
\Delta\left(V_{\alpha}^{ \pm}\right) & =\frac{1}{2} \alpha_{ \pm}^{2}(\alpha, \alpha)-\alpha_{0} \alpha_{ \pm}(\rho, \alpha)=1 \\
\alpha_{ \pm} & =\frac{\alpha_{0}}{2} \pm \sqrt{\left(\frac{\alpha_{0}}{2}\right)^{2}+1}
\end{aligned}
$$

Здесь мы подразумеваем, что $\alpha$ и $\beta$ - простые корни $s l_{3}$, а $\rho^{i}=(1 / 2) \sum_{+} \alpha^{i}-$ вектор Вейля, т.е. $(\alpha, \alpha)=2,(\alpha, \beta)=-1$ и $(\rho, \alpha)=(\rho, \beta)=1$. Рассмотрим алгебру

2 Теоретическая и математическая физика, т. 154, № 2, 2008 г. 
с двумя генераторами

$$
E_{\alpha}=\int d z: e^{i \alpha_{+}(\alpha, \varphi)}:, \quad E_{\beta}=\int d z: e^{i \alpha_{+}(\beta, \varphi)}:
$$

где контур интегрирования в комплексной плоскости $z$ проходит из точки $z=1$ вокруг нуля обратно в точку $z=1$ против часовой стрелки. Для произведения $n$ вершинных операторов получаем следующее выражение:

$$
\begin{aligned}
{\left[\left[V_{\alpha_{1}} \ldots V_{\alpha_{n}}\right]\right]:=} & \int_{\Gamma_{n}} d z_{1} \ldots d z_{n}: e^{i \alpha_{+}\left(\alpha_{1}, \varphi\right)}\left(z_{1}\right): \ldots: e^{i \alpha_{+}\left(\alpha_{n}, \varphi\right)}\left(z_{n}\right):= \\
= & \int_{\Gamma_{n}} d z_{1} \ldots d z_{n} \prod_{1 \leqslant k<l \leqslant n}\left(z_{k}-z_{l}\right)^{\alpha_{+}^{2}\left(\alpha_{k}, \alpha_{l}\right)} \times \\
& \times: e^{i \alpha_{+}\left(\alpha_{1}, \varphi\right)}\left(z_{1}\right) \ldots e^{i \alpha_{+}\left(\alpha_{n}, \varphi\right)}\left(z_{n}\right):
\end{aligned}
$$

где использовано правило Вика. Контур интегрирования $\Gamma_{n}$ (рис. 1) выбран согласно прескрипции Фельдера [6]. Он состоит из $n$ вложенных контуров единичного радиуса, ориентированных против часовой стрелки и идущих из точки $z=1$ вокруг нуля обратно в ту же точку. Интеграл (5), определенный таким образом, содержит сингулярности при $z_{k}=z_{l}$. Мы регуляризуем их с помощью раздвижки точек на малую величину $\varepsilon$; снятие регуляризации происходит в пределе $\varepsilon \rightarrow 0$.

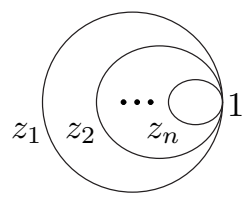

Рис. 1. Контур интегрирования $\Gamma_{n}$.

Генератор, соответствующий корню $\alpha+\beta$, дается следующим квантовым коммутатором:

$$
E_{\alpha+\beta}=-\left[E_{\alpha}, E_{\beta}\right]_{q^{-1}}:=-\left[\left[V_{\alpha} V_{\beta}-q^{-1} V_{\beta} V_{\alpha}\right]\right], \quad q=q_{+}=e^{i \pi \alpha_{+}^{2}} .
$$

Этот генератор не может быть записан как локальный интеграл в $z$-плоскости, он имеет некоторую нелокальную форму (см. раздел 3 ). Все остальные возможные $q$-коммутаторы $\left(E_{\alpha}, E_{\beta}\right.$ с $\left.E_{\alpha+\beta}\right)$ должны быть равны нулю согласно соотношениям Серра для $U_{q}\left(s l_{3}\right)$ :

$$
\left[E_{\alpha},\left[E_{\alpha}, E_{\beta}\right]_{q^{-1}}\right]_{q}=\left[\left[V_{\alpha} V_{\alpha} V_{\beta}-\left(q+q^{-1}\right) V_{\alpha} V_{\beta} V_{\alpha}+V_{\beta} V_{\alpha} V_{\alpha}\right]\right]=0 .
$$

Доказательство, приведенное в разделе 3, основано на регуляризации выражения (7) раздвижкой точек. Основное наблюдение в процессе доказательства (7) состоит в том, что все сингулярные по $1 / \varepsilon$ члены сокращаются во всех порядках.

Выражения (6), (7) определяют коммутационные соотношения для верхнетреугольной части квантовой группы $U_{q}\left(s l_{3}\right)$ с квантовым параметром $q_{+}$.

Существует классический предел данной конструкции $\alpha_{0} \rightarrow 0(q \rightarrow-1)$, при взятии которого действие (1) содержит только кинетический член. Этот предел не 
совпадает со стандартным пределом $q \rightarrow 1$, при котором квантовая группа переходит в соответствующую алгебру Ли. В данном случае при взятии классического предела мы получаем конструкцию Френкеля-Каца-Сегала [1], [2] в теории бозонной струны. Квантовый коммутатор (6) принимает вид $E_{\alpha} E_{\beta}-(-1)^{(\alpha, \beta)} E_{\beta} E_{\alpha}$, а множитель $(-1)^{(\alpha, \beta)}$ может быть удален с помощью введения коциклов в определение вершинных операторов (см. [2]).

\section{3. СООТНОШЕНИЯ СЕРРА ДЛЯ $U_{q}\left(s l_{3}\right)$}

В этом разделе мы приводим доказательство соотношений Серра (7).

Введем следующее обозначение для интеграла (5) с упорядоченным порядком интегрирования:

$$
I_{\alpha_{1} \ldots \alpha_{n}}=\int_{\arg z_{k}<\arg z_{k+1}-\varepsilon} d z_{1} \ldots d z_{n}: e^{i \alpha_{+}\left(\alpha_{1}, \varphi\right)}\left(z_{1}\right): \ldots: e^{i \alpha_{+}\left(\alpha_{n}, \varphi\right)}\left(z_{n}\right):,
$$

где $1 \leqslant k \leqslant n-1$. Можно явно построить третий генератор, используя $q=e^{i \pi \alpha_{+}^{2}}$ :

$$
\begin{aligned}
E_{\alpha+\beta} & =-\left[\left[V_{\alpha} V_{\beta}-q^{-1} V_{\beta} V_{\alpha}\right]\right]= \\
& =-\int_{\Gamma_{2}} d z_{1} d z_{2}\left(z_{1}-z_{2}\right)^{-\alpha_{+}^{2}}: e^{i \alpha_{+}(\alpha, \varphi)}\left(z_{1}\right) e^{i \alpha_{+}(\beta, \varphi)}\left(z_{2}\right):+q^{-1} \cdot(\alpha \leftrightarrow \beta) .
\end{aligned}
$$

При замене переменных $z_{1} \leftrightarrow z_{2}$ во втором интеграле возникает дополнительный компенсирующий множитель $q$ и контур интегрирования может быть заменен со-
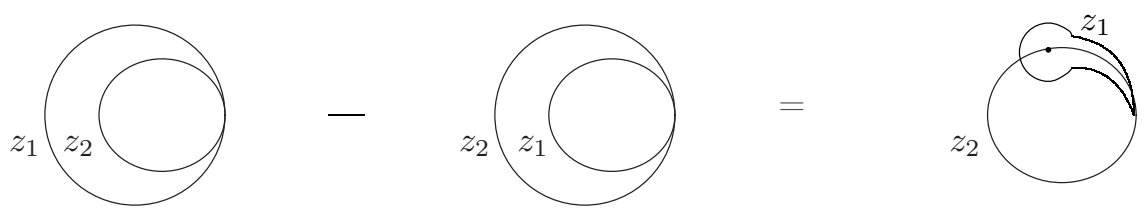

Рис. 2. Контуры интегрирования в формуле (9).

гласно рис. 2. Далее можно отдельно проинтегрировать по окружности радиуса $\varepsilon$ $\left(z_{1}-z_{2}=\varepsilon e^{i \psi}\right)$ и переписать интеграл в виде $(8)$ :

$$
\begin{aligned}
E_{\alpha+\beta}=- & \varepsilon^{1-\alpha_{+}^{2}} \int i d \psi d z_{2} e^{i\left(1-\alpha_{+}^{2}\right) \psi} \times \\
& \times: e^{i \alpha_{+}(\alpha, \varphi)}\left(z_{2}+\varepsilon e^{i \psi}\right) e^{i \alpha_{+}(\beta, \varphi)}\left(z_{2}\right):-\left(1-q^{-2}\right) I_{\alpha \beta} .
\end{aligned}
$$

Множитель $\left(1-q^{-2}\right)$ появился благодаря фактору $\left(z_{1}-z_{2}\right)^{-\alpha_{+}^{2}}$ в подынтегральном выражении. Заметим, что в классическом пределе $\alpha_{0} \rightarrow 0$ (или, что эквивалентно, $q \rightarrow-1)$ коэффициент при нелокальном члене $I_{\alpha \beta}$ обращается в нуль и мы воспроизводим классическое определение третьего генератора [1], [2]:

$$
E_{\alpha+\beta}=\int d z: e^{i(\alpha+\beta, \varphi)}:
$$

Главной проверкой верности нашей конструкции является выполнение тождества Серра (7) для квантовой группы $U_{q}\left(s l_{3}\right)$. Оно может быть переписано в следующем 
виде:

$$
\begin{aligned}
& {\left[\left[V_{\alpha} V_{\alpha} V_{\beta}-q^{-1} V_{\alpha} V_{\beta} V_{\alpha}\right]\right]-q\left[\left[V_{\alpha} V_{\beta} V_{\alpha}-q^{-1} V_{\beta} V_{\alpha} V_{\alpha}\right]\right]=} \\
& =\int_{C_{1}} d z_{1} d z_{2} d z_{3}\left(z_{1}-z_{2}\right)^{2 \alpha_{+}^{2}}\left(z_{1}-z_{3}\right)^{-\alpha_{+}^{2}}\left(z_{2}-z_{3}\right)^{-\alpha_{+}^{2} \times} \\
& \quad \times: e^{i \alpha_{+}(\alpha, \varphi)}\left(z_{1}\right) e^{i \alpha_{+}(\alpha, \varphi)}\left(z_{2}\right) e^{i \alpha_{+}(\beta, \varphi)}\left(z_{3}\right):- \\
& \quad-q \int_{C_{2}^{\prime}} d z_{1} d z_{2} d z_{3}\left(z_{1}-z_{2}\right)^{-\alpha_{+}^{2}}\left(z_{1}-z_{3}\right)^{2 \alpha_{+}^{2}}\left(z_{2}-z_{3}\right)^{-\alpha_{+}^{2} \times} \\
& \quad \times: e^{i \alpha_{+}(\alpha, \varphi)}\left(z_{1}\right) e^{i \alpha_{+}(\beta, \varphi)}\left(z_{2}\right) e^{i \alpha_{+}(\alpha, \varphi)}\left(z_{3}\right):
\end{aligned}
$$

Производя замену переменных $z_{1} \rightarrow z_{2}, z_{2} \rightarrow z_{3}, z_{3} \rightarrow z_{1}$ во втором интеграле (после которой контур $C_{2}^{\prime}$ заменяется на $C_{2}$ и появляется фактор $q^{-1}$ ) и интегрируя

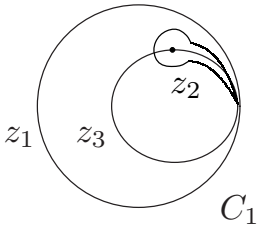

$C_{1}$

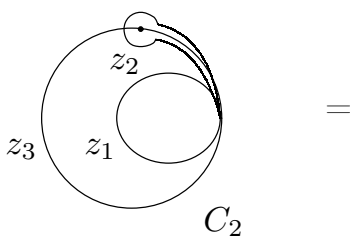

$C_{2}$

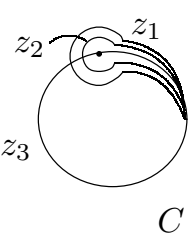

C

Рис. 3. Контуры интегрирования в формулах (11), (12) (во всех контурах направление движения против часовой стрелки).

по окружности радиуса $\varepsilon: z_{1}-z_{3}=\varepsilon e^{i \theta}, z_{2}-z_{3}=\varepsilon e^{i \psi}$, получаем для предыдущего выражения (см. рис. 3)

$$
\begin{aligned}
\varepsilon^{2} \int & i d \theta i d \psi d z_{3}\left(e^{i \theta}-e^{i \psi}\right)^{2 \alpha_{+}^{2}} e^{i\left(1-\alpha_{+}^{2}\right)(\theta+\psi)} \times \\
& \times: e^{i \alpha_{+}(\alpha \varphi)}\left(z_{3}+\varepsilon e^{i \theta}\right) e^{i \alpha_{+}(\alpha \varphi)}\left(z_{3}+\varepsilon e^{i \psi}\right) e^{i \alpha_{+}(\beta \varphi)}\left(z_{3}\right):+ \\
+ & \varepsilon^{1-\alpha_{+}^{2}} \int i d \psi d z_{1} d z_{3}\left(z_{1}-z_{3}-\varepsilon e^{i \psi}\right)^{2 \alpha_{+}^{2}}\left(z_{1}-z_{3}\right)^{-\alpha_{+}^{2}} e^{i\left(1-\alpha_{+}^{2}\right) \psi} \times \\
& \times: e^{i \alpha_{+}(\alpha \varphi)}\left(z_{1}\right) e^{i \alpha_{+}(\alpha \varphi)}\left(z_{3}+\varepsilon e^{i \psi}\right) e^{i \alpha_{+}(\beta \varphi)}\left(z_{3}\right):+ \\
+ & \varepsilon^{1-\alpha_{+}^{2}} \int i d \theta d z_{2} d z_{3}\left(z_{3}-z_{2}+\varepsilon e^{i \theta}\right)^{2 \alpha_{+}^{2}}\left(z_{2}-z_{3}\right)^{-\alpha_{+}^{2}} e^{i\left(1-\alpha_{+}^{2}\right) \theta} \times \\
& \times: e^{i \alpha_{+}(\alpha \varphi)}\left(z_{2}\right) e^{i \alpha_{+}(\alpha \varphi)}\left(z_{3}+\varepsilon e^{i \theta}\right) e^{i \alpha_{+}(\beta \varphi)}\left(z_{3}\right):+ \\
+ & \int_{C_{\varepsilon}} d z_{1} d z_{2} d z_{3}\left(z_{1}-z_{2}\right)^{2 \alpha_{+}^{2}}\left(z_{1}-z_{3}\right)^{-\alpha_{+}^{2}}\left(z_{2}-z_{3}\right)^{-\alpha_{+}^{2}} \times \\
& \times: e^{i \alpha_{+}(\alpha, \varphi)}\left(z_{1}\right) e^{i \alpha_{+}(\alpha, \varphi)}\left(z_{2}\right) e^{i \alpha_{+}(\beta, \varphi)}\left(z_{3}\right): .
\end{aligned}
$$

Первое слагаемое в (12), возникшее при интегрировании по двум окружностям радиуса $\varepsilon$, исчезает в пределе $\varepsilon \rightarrow 0$. Сингулярные слагаемые (второе и третье) взаимно сокращаются. Контур $C_{\varepsilon}$ в четвертом слагаемом совпадает с контуром $C$ за вычетом окружностей радиуса $\varepsilon$. Он содержит интегрирование по окружности $\left|z_{1}-z_{2}\right|=\varepsilon$, исчезающее в пределе $\varepsilon \rightarrow 0$. Оставшийся интеграл может быть переписан через базис интегралов $I_{\alpha_{1} \ldots \alpha_{n}}(8)$ :

$$
I_{\alpha \alpha \beta}+q^{2} q^{-4} I_{\alpha \alpha \beta}-q^{2} q^{-4} I_{\alpha \alpha \beta}-q^{-2} q^{2} I_{\alpha \alpha \beta}=0 .
$$


Это завершает доказательство тождеств Серра.

\section{4. КВАНТОВАЯ ГРУППА $U_{q}\left(\widehat{s l}_{2}\right)$}

Для обобщения приведенной конструкции на аффинный случай нужно ввести два дополнительных светоподобных поля $\varphi_{+}$и $\varphi_{-}$и заменить действие (1) на

$$
S=\int d^{2} z\left(\partial \varphi^{i} \partial \varphi^{i}+\partial \varphi_{+} \partial \varphi_{-}+i \alpha_{0} R\left(\rho^{i} \varphi^{i}+\rho_{+} \varphi_{-}+\rho_{-} \varphi_{+}\right)\right) .
$$

Пусть $\beta_{0}$ и $\beta_{1}-$ простые корни аффинной алгебры Ли $\widehat{s l}_{2}$ с матрицей Картана

$$
a_{i j}=\left(\begin{array}{cc}
2 & -2 \\
-2 & 2
\end{array}\right) .
$$

Вершинные операторы конформной размерности 1, соответствующие простым корням, задаются выражениями

$$
E_{0}=\int d z e^{i \alpha_{+}\left(\left(\beta_{0}, \varphi\right)+\varphi_{+}\right)}, \quad E_{1}=\int d z e^{i \alpha_{+}\left(\beta_{1}, \varphi\right)}
$$

Выпишем коммутационные соотношения $U_{q}\left(\widehat{s l}_{2}\right)$, содержащие только генераторы, соответствующие простым корням:

$$
\begin{gathered}
e_{\delta}=\left[E_{0}, E_{1}\right]_{q^{-2}}, \\
e_{n}=A(n, q)\left[E_{1},\left[E_{0},\left[E_{1},\left[E_{0}, \ldots\left[E_{1},\left[E_{0}, E_{1}\right]_{q^{-2}}\right] \ldots\right]_{q^{2}}\right]\right]_{q^{2}}\right], \\
e_{n \delta}=B(n, q)\left[E_{0}, e_{n-1}\right]_{q^{2}},
\end{gathered}
$$

где $A(n, q)$ и $B(n, q)$ - некоторые нормировочные множители. Генератор $e_{n}$ соответствует корню $\beta_{1}+n \delta$. Соотношения Серра в этом случае выглядят следующим образом:

$$
\begin{gathered}
{\left[e_{n+1}, e_{m}\right]_{q^{2}}+\left[e_{m+1}, e_{n}\right]_{q^{2}}=0,} \\
{\left[E_{1},\left[E_{1},\left[E_{1}, E_{0}\right]_{q^{2}}\right]\right]_{q^{-2}}=0, \quad m=n=0 .}
\end{gathered}
$$

Доказательство соотношений Серра (21) во многом аналогично случаю $U_{q}\left(s l_{3}\right)$ и содержится в приложении. Там же приведены формулы для генераторов (18), (19).

В классическом пределе $\left(\alpha_{0} \rightarrow 0\right)$ генераторы $(16)$ сводятся к

$$
E_{0}=\int d z e^{i\left(\left(\beta_{0}, \varphi\right)+\varphi_{+}\right)}, \quad E_{1}=\int d z e^{i\left(\beta_{1}, \varphi\right)},
$$

что совпадает с конструкцией Френкеля-Каца-Сегала для $\widehat{s l}_{2}$ [1], [2].

\section{5. ЗАКЛЮЧЕНИЕ}

Мы описали вертекс-операторную конструкцию $U_{q_{+}}\left(n_{+}\right)$, где $n_{+}-$борелевская подалгебра некоторой алгебры Ли $g$. Заметим, что аналогично можно построить $U_{q_{-}}\left(n_{-}\right)$. Открытой проблемой остается объединение двух частей в один объект.

Конструкции такого типа (использующие только скаляры $\varphi^{i}$ ) могут быть также построены для следующих алгебр: конечномерных простых типа $A, D, E$, аффинных $A^{(1)}, D^{(1)}, E^{(1)}$ и, возможно, некоторых гиперболических. 
Мы также обсудили связь между вертекс-операторной конструкцией квантовых групп и конструкцией Френкеля-Каца-Сегала. Возможные приложения результатов данной работы к квантованию конформной аффинной теории Тоды мы оставляем за рамками данной работы.

\section{ПРИЛОЖЕНИЕ}

Приведем доказательство тождеств Серра для $U_{q}\left(\widehat{s l}_{2}\right)$ и формулы для генераторов, соответствующих положительным корням $U_{q}\left(\widehat{s l}_{2}\right)$. Прежде всего, генератор, соответствующий нулевому корню (17), дается выражением

$$
\begin{aligned}
e_{\delta}=[ & \left.E_{0}, E_{1}\right]_{q^{-2}}=\varepsilon^{1-2 \alpha_{+}^{2}} \int i d \psi d z e^{i\left(1-2 \alpha_{+}^{2}\right) \psi} \times \\
& \times: e^{i \alpha_{+}\left((\beta 0, \varphi)+\varphi_{+}\right)}\left(z+\varepsilon e^{i \psi}\right) e^{i \alpha_{+}\left(\beta_{1}, \varphi\right)}(z):+\left(1-q^{-4}\right) I_{01} .
\end{aligned}
$$

В классическом пределе $\left(\alpha_{0} \rightarrow 0\right)$ второе слагаемое исчезает и первый интеграл сводится к $e_{\delta}=\int d z \partial \varphi e^{\varphi_{+}}$.

Доказательство тождеств Серра (21) сводится к доказательству следующего равенства:

$$
\begin{aligned}
& \int_{\Gamma} d z_{1} d z_{2} d z_{3} d z_{4}\left(z_{1}-z_{2}\right)^{2 \alpha_{+}^{2}}\left(z_{1}-z_{3}\right)^{2 \alpha_{+}^{2}}\left(z_{1}-z_{4}\right)^{-2 \alpha_{+}^{2}} \times \\
& \times\left(z_{2}-z_{3}\right)^{2 \alpha_{+}^{2}}\left(z_{2}-z_{4}\right)^{-2 \alpha_{+}^{2}}\left(z_{3}-z_{4}\right)^{-2 \alpha_{+}^{2} \times} \\
& \times: e^{i \alpha_{+}\left(\beta_{1}, \varphi\right)}\left(z_{1}\right) e^{i \alpha_{+}\left(\beta_{1}, \varphi\right)}\left(z_{2}\right) e^{i \alpha_{+}\left(\beta_{1}, \varphi\right)}\left(z_{3}\right) e^{i \alpha_{+}\left(\left(\beta_{0}, \varphi\right)+\varphi_{+}\right)}\left(z_{4}\right):=0 .
\end{aligned}
$$

Контур Г выбирается следующим образом. Каждая из переменных $z_{1}, z_{2}, z_{3}$ интегрируется от $z=1$ обратно до $z=1$ вокруг точки $z_{4}$, после этого переменная $z_{4}$ интегрируется вдоль единичной окружности. При этом контуры интегрирования вдоль $z_{1}, z_{2}, z_{3}$ вложены друг в друга.

Удобно ввести следующее обозначение. Пусть $\delta_{j}, 1<j<3$, обозначает контур интегрирования переменной $z_{j}$, состоящий из окружностей радиуса $\varepsilon$ вокруг $z_{4}$ : $z_{j}-z_{4}=\varepsilon e^{i \theta_{j}}$. Обозначим также через $\gamma_{j}$ контур интегрирования переменной $z_{j}$, состоящий из двух частей: сначала мы интегрируем против часовой стрелки вокруг дуги $0<\arg z_{j}<\arg z_{4}-\varepsilon,\left|z_{j}\right|>\left|z_{4}\right|$, затем по часовой стрелке вокруг дуги $0<\arg z_{j}<\arg z_{4}-\varepsilon,\left|z_{j}\right|<\left|z_{4}\right|$.

Согласно введенным обозначениям контур Г в (П.2) разделяется на восемь частей.

1. Интегрирование по $\delta_{1}, \delta_{2}, \delta_{3}$ и $z_{4}$. Этот интеграл пропорционален $\varepsilon^{3}$ и исчезает в пределе $\varepsilon \rightarrow 0$.

2 -4. Интегрирование по $\delta_{1}, \gamma_{2}, \gamma_{3}$ и $z_{4} ; \gamma_{1}, \delta_{2}, \gamma_{3}$ и $z_{4} ; \gamma_{1}, \gamma_{2}, \delta_{3}$ и $z_{4}$. Каждый из этих интегралов пропорционален интегралу

$$
\begin{aligned}
& \varepsilon^{2\left(1-\alpha_{+}^{2}\right)} \int i d \chi d x d y d z(x-y)^{2 \alpha_{+}^{2}}\left(x-z-\varepsilon e^{i \chi}\right)^{2 \alpha_{+}^{2}}(x-z)^{-2 \alpha_{+}^{2}} \times \\
& \quad \times\left(y-z-\varepsilon e^{i \chi}\right)^{2 \alpha_{+}^{2}(y-z)^{-2 \alpha_{+}^{2}} e^{i\left(1-2 \alpha_{+}^{2}\right) \chi} \times} \\
& \quad \times: e^{i \alpha_{+}\left(\beta_{1}, \varphi\right)}(x) e^{i \alpha_{+}\left(\beta_{1}, \varphi\right)}(y) e^{i \alpha_{+}\left(\beta_{1}, \varphi\right)}\left(z+\varepsilon e^{i \chi}\right) e^{i \alpha_{+}\left(\left(\beta_{0}, \varphi\right)+\varphi_{+}\right)}(z): .
\end{aligned}
$$

Здесь интегрирование по $x, y, z$ упорядочено согласно (8). Сумма коэффициентов пропорциональности равна нулю. 
$5-7$. Интегрирование по $\delta_{1}, \delta_{2}, \gamma_{3}$ и $z_{4}, \delta_{1}, \gamma_{2}, \delta_{3}$ и $z_{4}, \gamma_{1}, \delta_{2}, \delta_{3}$ и $z_{4}$. Эти интегралы аналогично взаимно сокращаются.

8. Интегрирование по $\gamma_{1}, \gamma_{2}, \gamma_{3}$ и $z_{4}$. Интегрирование по раздвинутым точкам $z_{1}$, $z_{2}$ и $z_{3}$ исчезает в пределе $\varepsilon \rightarrow 0$. Оставшийся интеграл может быть переписан в базисе $I_{\alpha_{1} \ldots \alpha_{n}}(10)$ и обращается в нуль аналогично (13).

Приведем также вычисления некоторых генераторов в аффинном случае. Они основаны на следующих формулах:

$$
\begin{aligned}
& {\left[E_{0}, I_{\epsilon_{0} \epsilon_{1} \ldots \epsilon_{n-1} \epsilon_{n}}\right]=\left(1-q^{-4}\right) \sum_{i=0}^{n}\left[a_{i}+1\right]_{q^{2}} I_{\epsilon_{0} \epsilon_{1} \ldots \epsilon_{i-1} 0 \epsilon_{i} \ldots \epsilon_{n-1} \epsilon_{n}},} \\
& {\left[E_{1}, I_{\epsilon_{0} \epsilon_{1} \ldots \epsilon_{n-1} \epsilon_{n}}\right]=\left(q^{2}-q^{-2}\right) \sum_{i=0}^{n}\left[b_{i}\right]_{q^{2}} I_{\epsilon_{0} \epsilon_{1} \ldots \epsilon_{i-1} 1 \epsilon_{i} \ldots \epsilon_{n-1} \epsilon_{n}},}
\end{aligned}
$$

где

$$
a_{i}=\frac{1}{2} \sum_{k=0}^{i-1}\left(\beta_{\epsilon_{k}}, \beta_{0}\right), \quad b_{i}=\frac{1}{2} \sum_{k=0}^{i-1}\left(\beta_{\epsilon_{k}}, \beta_{1}\right)
$$

и введено обозначение $I_{\epsilon_{0} \epsilon_{1} \ldots \epsilon_{n-1} \epsilon_{n}}=I_{\beta_{\epsilon_{0}} \beta_{\epsilon_{1}} \ldots \beta \epsilon_{n-1} \beta_{\epsilon_{n}}}\left(\epsilon_{i}=0\right.$ или 1$)$ (см. (8)).

Выражения для $e_{n}$ и $e_{n \delta}$ могут быть переписаны аналогично (5) и (12). Можно аналитически продолжить подынтегральные выражения (считая $\alpha_{+}^{2}$ комплексным, см. [15]) из области, в которой они хорошо определены, так что сингулярные по $\varepsilon$ слагаемые не дают вклада. Используя формулы, приведенные выше, можно переписать генераторы (18) в базисе интегралов $I_{\epsilon_{0} \epsilon_{1} \ldots \epsilon_{n-1} \epsilon_{n}}$ :

$$
e_{n}=A(n, q) q^{2 n}\left(1-q^{-4}\right)^{2 n} \sum_{\epsilon_{1}+\cdots+\epsilon_{2 n-1}=n} c_{0 \epsilon_{1} \ldots \epsilon_{2 n-1} 1} I_{0 \epsilon_{1} \ldots \epsilon_{2 n-1} 1},
$$

где суммирование идет по всем нетривиальным перестановкам $\epsilon_{1}, \ldots, \epsilon_{2 n-1}$. Коэффициенты $c_{\epsilon_{0} \epsilon_{1} \ldots \epsilon_{2 n-1} \epsilon_{2 n}}$ даются выражениями

$$
c_{\epsilon_{0} \epsilon_{1} \ldots \epsilon_{2 n-1} \epsilon_{2 n}}=\sum_{\substack{j_{1} \\\left(\epsilon_{j_{1}}=1\right)}} \sum_{\substack{j_{2} \neq j_{1} \\\left(\epsilon_{j_{2}}=0\right)}} \cdots \sum_{\substack{j_{2 n-1} \neq j_{2 n-2} \neq \cdots \neq j_{1} \\\left(\epsilon_{j_{2 n-1}}=1\right)}}\left[b_{j_{1}}\right]_{q^{2}}\left[a_{j_{2}}^{\prime}+1\right]_{q^{2}} \ldots\left[a_{j_{2 n-2}}^{\prime}+1\right]_{q^{2}}\left[b_{j_{2 n-1}}^{\prime}\right]_{q^{2}},
$$

где использованы обозначения

$$
a_{j_{2 l}}^{\prime}=\frac{1}{2} \sum_{\substack{k=0, k \neq j_{1}, \ldots, j_{2 l-1}}}^{j_{2 l}-1}\left(\beta_{\epsilon_{k}}, \beta_{0}\right), \quad b_{j_{2 l-1}}^{\prime}=\frac{1}{2} \sum_{\substack{k=0, k \neq j_{1}, \ldots, j_{2 l-2}}}^{j_{2 l-1}-1}\left(\beta_{\epsilon_{k}}, \beta_{1}\right) .
$$

Символ суммы $\sum_{j_{k}\left(\epsilon_{j_{k}}=1\right)}$ означает суммирование по всем $j_{k} \quad\left(1 \leqslant j_{k} \leqslant 2 n-1\right)$ таким, что $\epsilon_{j_{k}}=1$. Используя (19), (П.4), можно получить формулу для $e_{n \delta}$. Например, используя (П.4)-(П.7), получаем

$$
\begin{aligned}
& e_{\delta}=\left(1-q^{-4}\right) I_{01}, \\
& e_{1}=\left[E_{1},\left[E_{0}, E_{1}\right]_{q^{-2}}\right]=-q^{2}\left(1-q^{-4}\right)^{2} I_{011},
\end{aligned}
$$




$$
\begin{aligned}
e_{2 \delta}= & {\left[E_{0},\left[E_{1},\left[E_{0}, E_{1}\right]_{q^{-2}}\right]\right]_{q^{2}}=-q^{2}\left(1-q^{-4}\right)^{3}\left(I_{0101}+\left(1+[2]_{q^{2}}\right) I_{0011}\right) } \\
e_{2}= & {\left[E_{1},\left[E_{0},\left[E_{1},\left[E_{0}, E_{1}\right]_{q^{-2}}\right]\right]_{q^{2}}\right]=} \\
= & q^{4}\left(1-q^{-4}\right)^{4}\left(I_{01101}+\left(2+[2]_{q^{2}}\right) I_{01011}+\left(1+[2]_{q^{2}}\right)^{2} I_{00111}\right) \\
e_{3 \delta}=[ & {\left[E_{0},\left[E_{1},\left[E_{0},\left[E_{1},\left[E_{0}, E_{1}\right]_{q^{-2}}\right]\right]_{q^{2}}\right]\right]_{q^{2}}=q^{4}\left(1-q^{-4}\right)^{5}\left(\left(1+[2]_{q^{2}}\right)\left(2+[2]_{q^{2}}\right) I_{001101}+\right.} \\
& \quad+\left(3+[2]_{q^{2}}\right) I_{010101}+I_{011001}+\left(1+[2]_{q^{2}}\right)^{2}\left(2+[2]_{q^{2}}\right) I_{010011}+ \\
& +\left(1+[2]_{q^{2}}\right)\left(2+[2]_{q^{2}}+[2]_{q^{2}}\left(2+[2]_{q^{2}}\right)\right) I_{001011}+ \\
& \left.+\left(1+[2]_{q^{2}}\right)^{2}\left(1+[2]_{q^{2}}+[3]_{q^{2}}\right) I_{000111}\right)
\end{aligned}
$$

с точностью до нормировочных множителей $A(n, q), B(n, q)$.

Благодарности. Автор выражает признательность А. Герасимову за многочисленные полезные дискуссии, руководство и поддержку. Он также благодарен А. Александрову, В. Долгушеву, А. Дымарскому, А. Зотову, А. Конечному, В. Побережному и С. Харчеву за полезные обсуждения и Э. Ахмедову, А. Морозову и Е. Сусловой за ценные советы. Работа была частично поддержана грантом Президента РФ № 00-15-99296, РФФИ (грант № 07-02-00878), Программой поддержки ведущих научных школ (грант НШ-8004.2006.2), грантом INTAS № 00-334, а также Федеральным агенством по атомной энергии.

\section{Список литературы}

[1] I. Frenkel, V. Kac, Invent. Math., 62 (1980), 23; G. Segal, Comm. Math. Phys., 80 (1981), 301.

[2] P. Goddard, D. Olive, Internat. J. Modern Phys. A, 1 (1986), 303.

[3] M. B. Halpern, Phys. Rev. D, 12 (1975), 1684; 13 (1976), 337; T. Banks, D. Horn, H. Neuberger, Nucl. Phys. B, 108 (1976), 119.

[4] V. Dotsenko, Adv. Stud. Pure Math., 16 (1988), 123.

[5] A. Gerasimov, A. Marshakov, A. Morozov, M. Olshanetsky, S. Shatashvili, Internat. J. Modern Phys. A, 5 (1990), 2495.

[6] G. Felder, Nucl. Phys. B, 317 (1989), 215; Erratum, 324 (1989), 548.

[7] Б. Л. Фейгин, Э.В.Френкель, УМН, 43:5 (263) (1988), 227.

[8] A. Tsuchiya, Y. Kanie, Lett. Math. Phys., 13 (1987), 303.

[9] P. Bouwknegt, J. McCarthy, K. Pilch, Comm. Math. Phys., 131 (1990), 125.

[10] P. Bouwknegt, J. McCarthy, K. Pilch, Progr. Theoret. Phys. Suppl., 102 (1990), 67.

[11] A. Varchenko, V. Schechtman, Integral Representations of N-Point Conformal Correlators in the WZW Model, Preprint MPI/89-51, Max-Planck Institute, Bonn, 1989.

[12] A. Varchenko, Multidimensional Hypergeometric Functions and Representation Theory of Lie Algebras and Quantum Groups, Adv. Ser. Math. Phys., 21, World Scientific, Singapore, 1995.

[13] B. Feigin, E. Frenkel, "Integrals of motion and quantum groups", Integrable systems and quantum groups (Montecatini Terme, 1993), Lecture Notes in Math., 1620, Springer, Berlin, 1996, 349; arXiv: hep-th/9310022.

[14] O. Babelon, L. Bonora, Phys. Lett. B, 244 (1990), 220.

[15] V. Dotsenko, V. Fateev, Nucl. Phys. B, 240 (1984), 312; 251 (1985), 691.

Поступила в редакцию 16.01.2006, после доработки 8.08.2007 